\title{
Claude-Marie Bonnefont, commissaire du Directoire dans le département de la Vienne
}

Jean-Marie Augustin

\section{(2) OpenEdition}

1 Journals

\section{Édition électronique}

URL : https://journals.openedition.org/ahrf/781

DOI : 10.4000/ahrf.781

ISSN : 1952-403X

Éditeur :

Armand Colin, Société des études robespierristes

\section{Édition imprimée}

Date de publication : 1 décembre 2002

Pagination : 135-142

ISSN : 0003-4436

\section{Référence électronique}

Jean-Marie Augustin, «Claude-Marie Bonnefont, commissaire du Directoire dans le département de la Vienne ", Annales historiques de la Révolution française [En ligne], 330 | octobre-décembre 2002, mis en ligne le 17 avril 2008, consulté le 23 avril 2022. URL : http://journals.openedition.org/ahrf/781 ; DOI : https://doi.org/10.4000/ahrf.781 


\title{
CLAUDE-MARIE BONNEFONT, COMMISSAIRE DU DIRECTOIRE DANS LE DÉPARTEMENT DE LA VIENNE
}

\author{
JEAN-MARIE AUGUSTIN
}

\begin{abstract}
Claude-Marie Bonnefont, nommé en 1796, occupe les fonctions de commissaire du Directoire dans le département de la Vienne jusqu'au début du Consulat. Ce modéré, compétent et actif, se révèle un bon administrateur. Toujours présent lors des réunions de l'administration départementale, il est en relation étroite avec les municipalités de canton, accélère la conscription, veille à la sécurité des routes et lutte contre les « brigands royaux ». Dans les rapports qu'il adresse aux ministres, il rend compte de l'opinion publique dont il fait une présentation optimiste alors qu'il y a des signes d'agitation. Après le coup d'État du 18 Fructidor an V, Bonnefont devient la cible préférée des anciens Jacobins. II manque d'être destitué, mais réussit à se maintenir en place. Sous le Consulat et l'Empire, il sera secrétaire général de la préfecture de la Vienne.
\end{abstract}

Mots clés : administration ; Directoire ; commissaire du Directoire ; département ; Vienne ; municipalités de canton ; routes ; brigands royaux ; esprit public.

Aux termes de l'article 191 de la constitution de l'an III, «le Directoire exécutif nomme auprès de chaque administration départementale et municipale, un commissaire qu'il révoque lorsqu'il le juge convenable ». L'institution rappelle celle des procureurs établis par l'Assemblée constituante (1) auprès des administrations locales, dans la mesure où les commissaires sont pris parmi les citoyens domiciliés depuis un an dans le département, mais ils ne sont pas élus et ressemblent beaucoup plus aux agents nationaux de l'an II. Comme eux, ils sont désignés par l'autorité gouvernementale.

Le Directoire choisit avec le plus grand soin les commissaires centraux placés auprès des administrations départementales, en prenant l'avis des membres du Corps législatif. C'est sur la recommandation de Félix Faulcon,

(1) Th. DUCROCQ, «Les procureurs syndics de 1790 et les commissaires du Directoire exécutif de l'an III » et « Les procureurs syndics et les commissaires du Directoire exécutif dans le département de la Vienne », Congrès des sociétés savantes, section des sciences économiques et sociales, 1891, pp. 157-190. 
député de la Vienne au Conseil des Cinq-Cents (2), qu'est d'abord nommé, en frimaire an IV (novembre 1795), Jacques-Louis Pressac-Desplanches, précédemment agent national du district de Civray, mais celui-ci démissionne au bout de six mois, en prétextant l'éloignement de son domicile (3). Pour lui succéder, Faulcon qui, avec Thibaudeau et Creuzé-Latouche, exerce une influence déterminante dans le département, propose la candidature de Claude-Marie Bonnefont (4), lequel est nommé par arrêté du Directoire en date du 24 messidor an IV (12 juillet 1796) (5).

Le nouveau commissaire est né en 1751 à Autun, en Bourgogne, mais s'est marié en Poitou (6) dans une famille bourgeoise de Lusignan (7). En 1789, il exerçait les fonctions de notaire seigneurial et de feudiste à Chasseneuil (8), au nord de Poitiers. Comme il le dit lui-même, il est entré l'année suivante dans la carrière administrative, après avoir été élu au conseil général du département de la Vienne (9). Pendant un an, de septembre 1791 à octobre 1792, il est membre du directoire du département puis juge au tribunal de Lusignan (10) jusqu'en octobre 1793. Ayant été suspendu de ses fonctions par Ingrand, représentant en mission, il est incarcéré à la prison de la Trinité à Poitiers. Libéré après Thermidor, il est nommé administrateur du district de Lusignan, puis retrouve sa place, en l'an IV, au sein de l'administration centrale du département.

Bonnefont que même ses ennemis politiques s'accordent à reconnaître comme un homme compétent, intelligent, ponctuel et travailleur (11), demeure commissaire du pouvoir exécutif dans la Vienne pendant toute la période du Directoire. Il est en relation constante avec les cinquante et un commissaires placés auprès des municipalités de canton, en particulier Dassier, commissaire auprès de la municipalité de Poitiers (12).

(2) $\mathrm{AN}, \mathrm{F} / 1$ b II/Vienne 1, Lettre de Faulcon à Champagneux, secrétaire en chef du ministère de l'Intérieur.

(3) Grands notables du Premier Empire, ss dir. de Louis Bergeron et de Guy Chaussinand-Nogaret, t. 26,

Vienne, par Guillaume Lévêque, Emmanuel Dion et Sébastien Jahan, Paris, CNRS Éditions, 2000, pp. 233-236.

(4) AN, F/1 b II/Vienne 1, Lettre de Faulcon à Champagneux du 4 thermidor an IV (22 juillet 1796).

(5) AD Vienne, L 280, reg. 14, p. 46 verso, copie transcrite sur le registre des délibérations du conseil du département, 5 thermidor an IV (23 juillet 1796).

(6) Grands notables, t. 26, pp. 63-65.

(7) Vienne, arrondissement de Poitiers, chef-lieu de canton.

(8) Vienne, arrondissement de Poitiers, canton de Poitiers nord.

(9) AN, F/1 b 1/156/ 33, état de 1813.

(10) Pierre MASSÉ, «Les journées révolutionnaires à Lusignan », Bulletin de la Société des antiquaires de l'Ouest, $4^{\mathrm{e}}$ série., t. 1, 1949, pp. 213-250.

(11) AN, F/lbII/Vienne 2, Lettre de Motet, accusateur public, aux directeurs, 12 messidor an VII (30 juin 1799).

(12) Eugène DESGRANGES, « La centralisation républicaine sous le Directoire : Les municipalités de canton dans le département de la Vienne », Bulletin de la Société des antiquaires de l'Ouest, $4^{\mathrm{e}}$ sér., t. 2, 1954, pp. 569-627 et pp. 735-809 ; Roger DOUCET, «L'esprit public dans le département de la Vienne pendant la Révolution », Mémoires de la Société des antiquaires de l'Ouest, $3^{\mathrm{e}}$ sér., t. 11, 1908, pp. 262-409; Jean-Marie AUGUSTIN, La Révolution française en Haut-Poitou et Pays charentais, Toulouse, Bibliothèque historique Privat 1989, pp. 261-294 ; Jacques PÉRET, Histoire de la Révolution française en Poitou-Charentes, Poitiers, Projets Éditions, 1988, pp. 249-261. 
Suivant la fin de l'article 191, le commissaire «surveille et requiert l'exécution des lois ». Il est placé auprès des administrations locales pour les contrôler, rectifier les erreurs, stimuler l'ardeur des fonctionnaires, mais son rôle est bien supérieur à celui d'une surveillance ou d'une simple expédition du travail administratif. Il est « l'œil du gouvernement » et à ce titre, lui rend compte de tout ce dont il juge à propos de l'informer.

\section{La surveillance de l'administration}

La loi du 21 fructidor an III (7 septembre 1795) qui met en application le régime administratif fixé par la Constitution, fait au commissaire du pouvoir exécutif l'obligation d'assister, avec voix consultative, à toutes les délibérations de l'administration départementale. En complément, une circulaire du 27 frimaire an IV (18 décembre 1795), émanant du Directoire, estime qu'il « doit être présent à tout, il doit tout voir, tout connaître ».

Bonnefont remplit ses devoirs avec assiduité. Sa signature, attestant sa présence, est toujours apposée en marge du registre des séances (13). Il serait même plus diligent encore s'il en avait la possibilité, mais partout il se heurte à des administrateurs peu qualifiés, indociles ou apathiques. Dans les lettres qu'il adresse au Directoire, il se plaint des difficultés de sa fonction. « La correspondance de l'administration centrale avec les municipalités [écrit-il le 10 vendémiaire an VI ( $1^{\mathrm{er}}$ octobre 1796)] n'est pas soutenue. Celles-ci sont tellement insouciantes que les exhortations, les menaces mêmes ne peuvent vaincre leurs négligences » ou encore, dix jours plus tard : «Il est certain que presque tous les agents sont sans talents et sans moyens, qu'ils ne lisent pas les lois, qu'ils les conçoivent encore moins, qu'enfin l'insouciance est à son comble» (14). Dans une autre lettre, il écrit encore que «Les administrations municipales, soit par négligence, soit par ignorance, ne secondent pas les efforts de l'administration centrale pour l'exécution des lois; plusieurs commissaires [cantonaux] non seulement sont incapables, mais même n'ont pas la volonté de remplir leurs devoirs avec exactitude. Il faut un temps infini pour obtenir une réponse et le plus souvent elle est insuffisante, aussi peuton dire que les administrations civiles n'ont pas cette unité, cet ensemble nécessaire pour faire le bien » (15).

Chaque décade, Bonnefont adresse un rapport au ministre de l'Intérieur dans lequel il rend compte de la situation dans le département (16), mais le

(13) $\mathrm{AD}$ Vienne, L 280 à 285, reg. 15 à 19; son nom s'écrivait indifféremment Bonnefond ou Bonnefont mais lui-même signait plus volontiers Bonnefont avec un t.

(14) Ibid., L 35, compte rendu au ministre de l'Intérieur.

(15) Ibid., compte rendu au ministre de l'Intérieur du 1" nivôse an VI (21 décembre 1797).

(16)Ibid., L 35 à 37. 
commissaire central ne se contente pas de faire remonter l'information. Il propose des solutions qui pourraient résoudre les difficultés. Puisque les commissaires cantonaux sont peu instruits et négligents, il conviendrait de les remplacer par des fonctionnaires pris indistinctement dans tous les départements et convenablement rémunérés. Afin de donner plus d'action à la partie administrative et de diminuer les dépenses, il préconise aussi de réduire le nombre des cantons (17). Lorsque le Directoire décide, par mesure d'économie, de supprimer la gratuité du courrier entre les municipalités et renvoie les frais de port sur les sous-additionnels payés par les contribuables, Bonnefont constate que les administrateurs s'abstiennent d'aller chercher les lettres aux relais de poste et ne répondent pas : «tout reste dans un état de stagnation effrayant », écrit-il, et il craint que la correspondance ne soit interrompue si on n'y remédie pas promptement (18).

La préoccupation majeure du commissaire central est cependant relative à l'état d'insécurité permanente qui affecte le département. Des bandes de déserteurs errent dans les campagnes. Les pillages se multiplient. Dans les cantons proches des Deux-Sèvres, des mendiants armés se font remettre du blé et de l'argent par la menace et la violence. Plus grave, des bandes de « chauffeurs » attaquent les métairies isolées et terrorisent les paysans, avant de se volatiliser dans les forêts voisines. À partir de l'an VI apparaissent des brigands d'un genre nouveau qui attaquent les diligences sur la route de Paris à Bordeaux et sur son embranchement en direction de La Rochelle (19). Ce n'est pas que les attaques de voitures publiques soient nouvelles, mais les voleurs se font remarquer par l'élégance de leurs vêtements, la qualité de leurs chevaux et la distinction de leurs manières. En outre, ils ne font aucun mal aux voyageurs et déclarent ne vouloir s'en prendre qu'à l'argent du gouvernement contenu dans les bagages. La rumeur fait alors état de «brigands royaux» qui pillent les fonds publics afin de recueillir l'argent nécessaire à la reprise de l'insurrection vendéenne. Bien qu'aucune preuve ne soit établie dans ce sens, la proximité de la Vendée conduit le commissaire central à faire l'amalgame avec la recrudescence des vols de diligence.

Contre ces «brigands royaux », l'administration départementale fait marcher des brigades de gendarmerie et les colonnes mobiles formées de gardes nationaux qui leur servent d'auxiliaires. Des battues sont organisées dans les bois situés aux environs des lieux où les diligences ont été pillées.

(17) Sous le Directoire, le district est supprimé et les cantons sont dotés d'une organisation municipale, sauf dans les communes de plus de 5000 habitants qui conservent leur municipalité. Le nombre des cantons a été réduit au début du Consulat; en même temps, le district est rétabli sous le nom d'arrondissement et le canton, privé de son administration, redevient une simple unité électorale et judiciaire.

(18) Arch. dép. Vienne, L 35, compte rendu au ministre de l'Intérieur du 11 pluviôse an VI (30 janvier 1798).

(19) Jean-Marie AUGUSTIN, «Les brigands royaux», Les grandes affaires criminelles de Poitiers, Mougon, Geste Éditions, 1995, pp. 123-139. 
Des visites domiciliaires sont effectuées chez des particuliers soupçonnés d'être complices, mais sans succès. Les brigands sont introuvables.

Bonnefont rend compte des faits au ministre de la Police générale (20). À titre préventif, des patrouilles sont établies pour éclairer les routes et escorter les voitures, mais elles ne sont pas assez nombreuses et les gardes nationaux qui les composent manquent de fusils et de munitions. Faute de moyens suffisants en hommes et en armes, le commissaire central est impuissant à venir à bout du brigandage. L'insécurité demeure sur les routes du département et l'inquiétude grandit dans la mesure où ces attaques de diligences ont vraisemblablement une inspiration politique.

\section{L'œil du gouvernement}

Dans ses rapports adressés au Directoire ou aux ministres, Bonnefont fait état des opinions modérées ou pour le moins attentistes des habitants du département de la Vienne. Il évoque «la très grande majorité » du peuple, éprouvant «un peu d'indifférence, beaucoup d'égoïsme, fatigué des intrigues, des mouvements, ne soupirant que pour le repos, désirant le règne des lois » (21). Il se plaint de ce que «les citoyens sont dociles, crédules, enclins au repos et à la tranquillité, indolents, insouciants, timides, craintifs et faciles à devenir le jouet des factieux et des charlatans » (22).

Il y a pourtant des signes qui dénotent une certaine agitation. Les catholiques s'enhardissent à fréquenter les messes clandestines célébrées par les prêtres réfractaires (23). Des agents dévoués à la cause de la monarchie fomentent des troubles dans les campagnes, excitent les paysans à désobéir aux lois et encouragent les jeunes gens à refuser la conscription. Certains cantons, dans la région de Loudun (24) et aux confins des départements de l'Indre et de l'Indre-et-Loire (25), ne sont pas sûrs. Dans une quinzaine de communes du département les arbres de la Liberté ont été coupés. À Poitiers, des habitants connus pour leurs opinions jacobines ont trouvé des potences peintes sur la porte de leurs maisons (26).

(20) AD Vienne, L 17 à 19.

(21) AN, F/lcIII/Vienne 5 Rapport de Bonnefont au ministre de l'Intérieur du $1^{\mathrm{er}}$ messidor an VI (19 juin 1798).

(22) Ibid, Rapport de Bonnefont au ministre de l'Intérieur du 12 vendémiaire an VII (3 octobre 1798).

(23) [bid., F/7 259, Lettre de Bonnefont au ministre de la Police du 7 prairial an V (26 mai 1797).

(24)Ibid., F/lcIII/Vienne 1, Protestation des «patriotes de 89» de Loudun au Directoire, du 20 brumaire an IV (11 novembre 1795).

(25) Ibid., F/7 289, Enquête faite par 29 patriotes du département de la Vienne sur les agissemements des contre-révolutionnaires, comprenant 38 dénonciations réunies entre le 18 fructidor an V (4 septembre 1797) et le 6 frimaire an VI (26 novembre 1797).

(26) Ibid., F/lblI/Vîenne 2, Pétition des républicains de Poitiers au ministre de l'Intérieur du 6 messidor an VII (24 juin 1799). 
Après le coup de force anti-royaliste du 18 fructidor an V (4 septembre 1797), les sanctions tombent. Le président Chasteau et un membre de l'administration départementale sont révoqués par le Directoire exécutif (27). Soixante-dix suspensions ou destitutions frappent des administrateurs ou des commissaires dans les municipalités de canton (28). Les mesures de révocation qui ont été prévues par la constitution pour des raisons administratives sont prises ici à des fins politiques.

Cette épuration confirme la victoire des modérés qui soutiennent le régime. Bonnefont écrit au ministre de l'Intérieur : «Il y a trois partis bien prononcés dans ce département : les royalistes avec les fanatiques, les anarchistes et les vrais républicains... le troisième a combattu et contenu alternativement les deux premiers » (29). Avec beaucoup d'optimisme, le commissaire central continue à faire une présentation satisfaisante de l'opinion publique. Dans toutes les occasions, notamment après la célébration des fêtes républicaines, il constate qu'un attachement de la très grande majorité des citoyens s'est manifesté à l'égard du gouvernement. Les cérémonies s'accomplissent au milieu d'un grand concours du peuple, animé du plus vif enthousiasme (30). De son côté, l'administration départementale remarque, après la fermeture du Cercle constitutionnel fréquenté par les Jacobins, que « la masse du peuple parait avoir abandonné les agitateurs et se porte vers les autorités constituées » (31).

Il y a bien cependant un retour des anciens « terroristes » évincés après Thermidor : Bernazais, Planier, ancien président du tribunal criminel de la Vienne (32), Fradin, Jean Alexandre et Dardillac. Ils trouvent en Motet qui s'est maintenu dans les fonctions d'accusateurs public, un porte-parole pour dénoncer les agissements des royalistes.

Bonnefont devient l'une de leurs cibles préférées. Un factum intitulé « Reproches à faire aux administrateurs de la Vienne », lui fait grief d'avoir profité de sa situation pour acheter des biens nationaux à bas prix. Il fréquenterait aussi à Poitiers, en compagnie d'autres notables, une académie de jeux considérée comme la «peste de la commune » (33). Plus graves sont les accusations de connivence avec la Contre-Révolution. Le 18 thermidor an VI (5 août 1798), une dénonciation signée d'un républicain, l'accuse de compli-

(27) AD Vienne, L 281, reg. des délibérations 15, p. 144, copie de l'arrêté du Directoire exécutif du 15 brumaire an VI (5 novembre 1797).

(28) Eugène DESGRANGES, art. cité, pp. 611-613.

(29) AN, F/lcIII/Vienne 5,10 vendémiaire an VI (1« octobre 1797).

(30) Roger DOUCET, op. cit., p. 334.

(31) AN, F/7 328, Lettre au ministre de la Police du 29 pluviôse an VI (17 février 1798).

(32) Pierre MASSÉ, «La déportation de Planier », Bull, de la Société des antiquaires de l'Ouest, $3=$ sér. t. 12,1939 , pp. 205-229.

(33) AN, F/1 blI/Vienne 2. 
cité avec les émigrés et les prêtres réfractaires (34). L'année suivante, les pétitions affluent pour demander sa révocation (35).

La réprobation englobe le commissaire central et les membres modérés de l'administration départementale. Dans une lettre écrite au ministre de la Police générale, le 22 fructidor an VII (8 septembre 1799), Motet les dénonce ensemble, en leur imputant la responsabilité des actes commis contre l'autorité publique : arbres de la Liberté arrachés, pamphlets séditieux, retour des prêtres et des religieuses à Poitiers, cris de «Vive le roi », menaces contre les patriotes et les acquéreurs de biens nationaux, réunions clandestines d'émigrés rentrés, manœuvres destinées à encourager les conscrits à déserter, etc. Tout cela cesserait ajoute-t-il, si Boncenne, membre influent de l'administration centrale, et Bonnefont étaient destitués (36). Pour le remplacement du commissaire, Motet avait déjà proposé aux Directeurs les noms de trois jacobins et en premier lieu, celui de Dardillac (37).

La démarche n'a de chance d'aboutir que si elle est appuyée par la députation de la Vienne. Or, tous les membres poitevins du Corps législatif protègent le commissaire central. Dès lors, la demande de révocation est présentée par les députés d'Indre-et-Loire : Japhet, Texier-Olivier, Chalmel et Grizol, sous prétexte que les désertions de conscrits, tolérées par Bonnefont auraient des répercussions dans leur département. Ils proposent également Dardillac pour le remplacer (38). Les députés de la Haute-Vienne se joignent à eux pour réclamer son départ, mais ils préfèrent la nomination à sa place de l'ancien conventionnel Ingrand qui avait été représentant en mission dans le département de la Vienne sous la Terreur (39).

À Paris, le gouvernement hésite : «Le département de la Vienne [écrit le ministre de la Police générale au ministre de l'Intérieur] a offert jusqu'à présent un aspect tranquille, mais cet état est-il dû au bon esprit qui y règne, à la sagesse et à l'énergie des autorités, ou bien est-il le résultat d'une administration molle et sans énergie qui a acheté, par une condescendance dangereuse, une tranquillité précaire ? Le Directoire doit-il être rassuré sur ce département ou bien doit-on le considérer comme renfermant les éléments de la rébellion dont le développement peut s'opérer d'un moment à l'autre ?» (40) Le ministre de l'Intérieur fait part de la même inquiétude à son collègue de la Guerre : «Si j'en crois divers rapports, ce département n'est pas en harmonie

(34) Ibid., F/3 696, Lettre de Giraud au ministre de la Police.

(35) Ibid., F/1 Ml/Vienne 2, Pétition anonyme dont l'auteur se révélera être le président du canton de Dissay (arr. de Poitiers, canton de Saint-Georges-les-Baillargeaux) dans une lettre du 17 thermidor an VII (4 août 1799) et pétition des « Républicains de Poitiers » du 10 thermidor an VII (28 juillet 1799).

(36) Ibid.

(37) Ibid, Lettre du 12 messidor an VII (30 juin 1799).

(38) Ibid., Lettres du 26 messidor, 15 et 23 fructidor an VII (14 juillet, $1^{\text {er }}$ et 9 septembre 1799). août 1799).

(39) Ibid., Rapport présenté au Directoire par le ministre de l'Intérieur, en thermidor an VII (juillet-

(40) Ibid., 25 fructidor an VII (11 septembre 1799). 
avec le système républicain et la faute paraît en être au commissaire central dont on peint le civisme comme au moins douteux » (41). La réponse du ministre de la Guerre se veut rassurante sur la question essentielle de la conscription : "Les levées des conscrits [écrit-il] se sont faites avec assez de succès dans ce département. Quelques désertions il est vrai ont eu lieu, mais au moyen des mesures rigoureuses et énergiques prises par l'administration centrale, tant pour l'arrestation des fuyards que pour leur renvoi aux différents corps, la grande majorité a rejoint l'armée. La conduite de M. Bonnefond est également digne d'éloge, il a constamment apporté dans cette opération tout le zèle, l'exactitude et le patriotisme qu'elle exigeait » (42).

Le commissaire central a subi de nombreuses attaques, mais le gouvernement le maintient jusqu'après le 18 Brumaire. Cet homme modéré, très compétent et assidu au travail, est parfaitement apte à remplir des tâches administratives. Il n'a pas cependant le caractère assez fort pour en imposer aux factions politiques. C'est pourquoi, au moment de la nomination des préfets, il n'est pas retenu à la différence de Jean-Philippe Maret commissaire en Côte d'Or, nommé dans le Loiret, ou de Claude-Antoine Marson, commissaire en Loire-Inférieure, nommé dans le Doubs (43).

C'est Cochon de Lapparent ancien conventionnel et ministre de la Police sous le Directoire, qui devient préfet de la Vienne (44). Bonnefont n'a ni la notabilité, ni l'étoffe pour remplir une telle fonction. Toutefois ses qualités de gestionnaire sont retenues. Le 6 floréal an VIII (26 mai 1800), il est nommé secrétaire général de la préfecture de la Vienne (45), et le restera pendant le Consulat et l'Empire. Sa connaissance du terrain fournit une aide précieuse aux trois préfets qui se sont succédés durant cette période dans le département. Destitué en 1814, il redevient secrétaire général de la préfecture sous les Cent-Jours, avec en outre la fonction de sous-préfet à Civray. Le retour du roi entraîne à nouveau sa révocation, mais Louis XVIII consent à lui accorder une pension de retraite. Il est mort à Poitiers, en 1833.

\author{
Jean-Marie AUGUSTIN \\ Université de Poitiers \\ Faculté de droit et des sciences sociales \\ 93, avenue du recteur Pineau \\ 86022 Poitiers
}

(41) Ibid., Lettre de fructidor an VII (septembre 1799).

(42) Ibid., Lettre au ministre de l'Intérieur du 4 vendémiaire an VIII (26 septembre 1799).

(43) René BARGETON, Pierre BOUGARD, Bernard LE CLERE et Pierre-François PINAUD, Les préfets du 11 ventôse an VIII au 4 septembre 1870, Paris, Archives nationales, 1981, pp. 209 et 212.

(44) Paul BOUCHER, Charles Cochon de Lapparent, conventionnel, ministre de la Police, préfet de l'Empire, Paris, A et J. Picard, 1969 ; Grands notables, t. 26, Vienne, pp. $89-91$; Description générale du département de la Vienne par Charles COCHON DE LAPPARENT, texte annoté par Jean-Marie AUGUSTIN, La Crèche, Geste éditions et Faculté de droit et des sciences sociales de Poitiers, 2000.

(45) Arch, nat., F/lbl/156/33, état de 1813. 